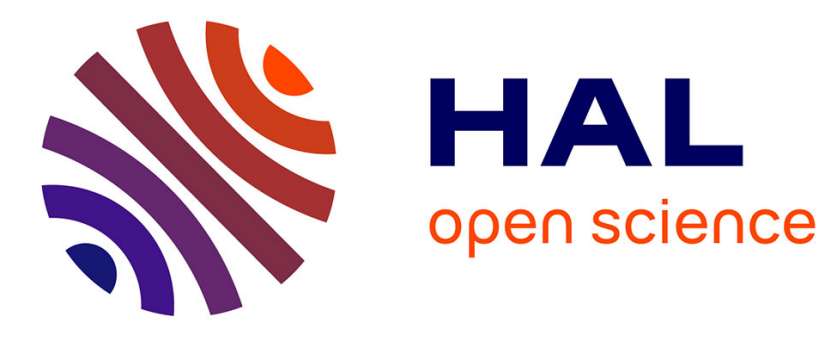

\title{
Immigration et héritage colonial
}

\author{
Abdellali Hajjat
}

\section{To cite this version:}

Abdellali Hajjat. Immigration et héritage colonial. Marie Poinsot; Serge Weber. Migrations et mutations dans la société française. État des savoirs, La Découverte, pp.257-265, 2014. halshs01059654

\section{HAL Id: halshs-01059654 \\ https://shs.hal.science/halshs-01059654}

Submitted on 31 May 2017

HAL is a multi-disciplinary open access archive for the deposit and dissemination of scientific research documents, whether they are published or not. The documents may come from teaching and research institutions in France or abroad, or from public or private research centers.
L'archive ouverte pluridisciplinaire HAL, est destinée au dépôt et à la diffusion de documents scientifiques de niveau recherche, publiés ou non, émanant des établissements d'enseignement et de recherche français ou étrangers, des laboratoires publics ou privés. 
Abdellali Hajjat

La question de l' " héritage colonial » fait l'objet d'un débat houleux au sein des sciences sociales à partir de l'émergence en 2003-2005 d'une controverse publique sur le «fait colonial » [Bertrand, 2006 ; Coquery-Vidrovitch, 2009]. Celle-ci se situe à l'intersection de plusieurs espaces sociaux (champ politique, espace des mobilisations et champ scientifique), disciplines académiques (histoire, science politique, sociologie, anthropologie et études littéraires) et espaces nationaux. Les oppositions très tranchées, laissant une faible place à un débat scientifique serein, ont révélé des clivages institutionnels au sein de l'université, des oppositions théoriques, ainsi qu'une lutte symbolique pour le monopole de la référence coloniale. Une sociologie de cette controverse reste à écrire, qui mettrait en relation les positions, les trajectoires et les prises de position des protagonistes, dont certains circulent facilement d'un espace social à un autre.

La controverse strictement scientifique soulève de multiples questions théoriques et méthodologiques, allant de la non-pertinence des cadres d'analyse proposés par les postcolonial studies à la non-prise en compte de l'histoire coloniale dans l'écriture de l'histoire nationale, en passant par l'autonomie de la recherche scientifique à l'égard des catégories et sollicitations de l'espace public. Il ne s'agit pas ici d'étudier toutes les dimensions des débats scientifiques [Weil, Dufoix, 2005 ; Bancel, Blanchard, Lemaire, 2005 ; Guénif-Souilamas, 2006 ; Smouts, 2007 ; Bancel et al., 2010], mais de focaliser l'attention sur la problématique des continuités et des ruptures entre le passé de la situation coloniale et le présent de la situation de l'immigration postcoloniale. 
En 1998, les historiens de l'Association pour la connaissance de l'histoire de l'Afrique contemporaine (ACHAC) publient un petit livre au titre évocateur, De l'indigène à l'immigré, qui traite du rapport entre passé colonial et présent de l'immigration à travers les représentations dominantes de l'Arabe et du Noir. Leurs préoccupations rencontrent celles de certains historiens de la colonisation et de l'immigration qui se désolent, à juste titre, des lacunes de l'histoire des immigrations maghrébine et africaine et envisagent l'immigration, la colonisation et le racisme sous le prisme d'une « histoire liée » [Liauzu, 2000].

Pour comprendre le succès des représentations et exhibitions coloniales, notamment sous la III République, les historiens de l'ACHAC utilisent le concept de "culture coloniale » sans vraiment le définir. Précisément, cet effort de définition «n'est pas l'objet de [leur] démarche tant la notion de culture de masse est déjà complexe » et ils se résignent seulement à " en cerner les contours " [Blanchard, Lemaire, 2003, p. 8]. En ce sens, la culture coloniale est, selon eux, " ce qui fait notre différence, issue de l'histoire, dans notre relation aux autres, aux ex-colonies et au monde, par rapport aux autres puissances occidentales, coloniales ou pas » [ibid.].

Le manque de définition claire et circonscrite de la « culture coloniale » fait écho à une des pierres d'achoppement de la controverse scientifique sur l'héritage colonial, qui porte sur la pertinence du concept de "fracture coloniale " pour comprendre la réalité de la société française. Là encore, comme le reconnaissent les promoteurs de la formule, la définition "n'est pas chose aisée » [Bancel, Blanchard, Lemaire, 2005, p. 13]. Tout en ne prétendant pas « réduire l'éclairage de la société française contemporaine aux seules lumières des articulations colonisation/ postcolonie [...], ni d'ailleurs invoquer à ce propos un héritage linéaire, une reproduction à l'identique des pratiques du passé » [ibid., p. 12], ce concept « voudrait à la fois signifier la tension et les effets de la postcolonialité : il recouvre des réalités multiples et des situations hétérogènes, dans la mesure où ces réalités et ces situations peuvent être éclairées, en partie, par des processus de longue durée, reliés à la situation coloniale " [ibid., p. 13].

Or la démonstration du livre n'a pas convaincu plusieurs historiens de la colonisation, notamment C. Liauzu, D. Hemery, G. Meynier et P. Vidal-Naquet, qui ont réagi dans un article de Libération daté du 16 novembre 2005 et considèrent que la " fracture coloniale " est une «formule aussi creuse et raccrocheuse que la fracture sociale » et une manière d'occulter « la réalité de conflits sociaux et de mouvements qu'il faut analyser et auxquels il faut apporter des réponses ». Par ailleurs, les historiens, politistes et sociologues d'inspiration bourdieusienne ou marxiste expriment des réticences à l'égard du concept de « fracture 
coloniale ", en raison du risque d'occultation de la dimension de classe de la condition de l'immigration postcoloniale.

pour autant, l'hypothèse de l'inhérence de l'expérience coloniale n'est pas en sol " intrinsèquement erronée " [Bayart, Bertrand, 2006]. Mais "elle demande à être démontrée au cas par cas plutôt que d'être posée en substantif et en postulat quasi métaphysique » [ibid.]. Par conséquent, le débat réside moins dans la pertinence de la problématique que dans la réponse à apporter à la question suivante : comment le passé colonial agit-1l dans le présent de l'immigration postcoloniale?

\section{Situation coloniale, situation postcoloniale}

Dès le début des années 1980, le sociologue Abdelmalek Sayad a été l'un des premiers chercheurs à soulever ce problème à partir d'une sociologie de l'immigration algérienne. Tout d'abord, il considère qu'il est difficile pour l'immigration postcoloniale de rompre avec le passé, parce que « les relations coloniales anciennes continue(nt) à peser sur le sort de cette immigration, sur son statut, sur son importance relative, sur sa place au sein de la société ainsi que sur les réactions à son égard toujours marquées de la signification que leur imprime l'histoire coloniale " [Sayad, 1982, p. 42]. Il envisage ainsi la « présence du passé » colonial en termes de structures et de hiérarchies sociales : la condition de l'immigré postcolonial (ou plutôt celle d' " ex-colonisé » ou d' " issu des anciennes colonies ", pour reprendre sa terminologie) doit être inscrite dans une histoire sociale longue, où les rapports de domination produisent des effets d'hystérésis bien après l'avènement des indépendances. Il démontre qu'il existe une homologie de position entre le colonisé et l'immigré postcolonial, mais il souligne aussi la différence de nature entre la situation coloniale et celle de l'immigration postcoloniale.

En effet, il considère qu' ' aussi éclairante que soit la comparaison entre les deux situations [...], elle ne saurait masquer la différence essentielle, différence de nature qui sépare les deux cas de figure » [Sayad, 1994, p. 10]. Alors que, dans la situation coloniale, c'est la société " assimilatrice " qui s'est imposée par la force sur le territoire des colonisés, dans la situation de l'immigration postcoloniale, c'est la population « à assimiler » qui s'est installée sur le territoire métropolitain. Ici, la question du territoire est essentielle, parce que, si les contradictions coloniales sont " résolues " par le nationalisme anticolonialiste, l'irrédentisme est tout simplement inconcevable pour l'immigration postcoloniale.

La perspective nuancée de Sayad l'amène à mobiliser la notion $\mathrm{d}^{\prime}$ " exception ". Selon lui, " d'une certaine manière, l' "exception coloniale" se continue dans l'immigration et par l'immigration qui 
comporte elle aussi ses exceptions » [Sayad, 1994, p. 28]. Il s'agit d'analyser la reproduction transformée d'un traitement social d'exception dans le sort réservé à une population passée du statut d'indigène à celui de ressortissant d'un pays étranger et/ou à celui de citoyen français. Autrement dit, même si l'exception n'est pas légitimée juridiquement comme dans le droit colonial, elle persiste dans la société postcoloniale au travers de processus sociaux de hiérarchisation et d'infériorisation. Le traitement différentiel entre les immigrations postcoloniales et les immigrations européennes, qui ont aussi fait l'objet de violences physiques et de discriminations à leur arrivée [Noiriel, 2008], ne réside pas dans leur supposée différence de «nature ", mais dans l'histoire des relations sociales inscrites, sans s'y réduire, dans le sillage des rapports coloniaux.

En ce sens, plusieurs travaux ont étudié la construction d'une " altérité de l'intérieur " [Guénif-Souilamas, 2007, p. 344-352] à travers une analyse des discours publics sur les populations arabes, musulmanes et/ou noires de France. De ce point de vue, "dans un parallélisme parfait, les mots qui s'appliquent alors aux indigènes désignent les immigrants successifs et plus particulièrement les "presque Français" venus des anciennes colonies. [...] Comme hier l'indigène, l'immigrant - et son descendant -, surtout s'il est musulman, est aujourd'hui prisonnier de sa nature " [Guénif-Souilamas, in Bancel et al., 2005, p. 203]. Au travers d'une histoire de longue durée des discours publics sur l'immigration, certains travaux insistent sur le " tournant colonial " [Noiriel, 2008, p. 483]. Ils soulignent le fait que la guerre d'Algérie constitue un " moment de rupture " parce qu'elle participe au renouveau du discours sur l'inassimilabilité des immigrés. Mais la question de l'assimilation des immigrés n'est pas spécifique aux immigrations postcoloniales puisqu'elle se posait au XIX ${ }^{e}$ siècle de manière simultanée en métropole et aux colonies, pour les métropolitains "non assimilés ", les indigènes et les immigrés européens. Cependant, on observe une divergence dans les discours dominants sur l'assimilation : tandis qu'on considère les étrangers européens comme assimilables, l'inassimilabilité des indigènes des colonies devient un dogme fondé sur la construction d'une différence raciale irréductible. Aux XIx ${ }^{e}$ et $\mathrm{xx}^{\mathrm{e}}$ siècles, le discours républicain sur l'assimilation des Européens considère ainsi que celle-ci est " possible ", parce que les Européens sont des " frères de race ", tandis que les indigènes font partie des " races exotiques ». Or le discours sur l'inassimilabilité des populations ex-colonisées se perpétue après les indépendances, le vocabulaire culturel ou ethnique se substituant au vocabulaire racial [Hajjat, 2012].

La guerre d'Algérie participe aussi à la construction d'un « ennemi intérieur », notamment musulman. Les représentations dominantes du musulman doivent de fait beaucoup aux représentations construites 
durant la période coloniale [Hajjat, Mohammed, 2013]. Une analyse de la presse écrite et télévisée des années 1970 à 2004 permet de « retracer les étapes de l'affirmation progressive des oppositions manichéennes véhiculées par les discours médiatiques associés aux images de $I^{\prime \prime}$ Arabe" $»$. Ces fractures, opposant vrais et faux Français, bons et mauvais immigrés, islam modéré et islam radical, assimilable ou inassimilable, menaçant ou non, se sont incarnées dans la mise en scène médiatique de personnages stéréotypés, devenus récurrents depuis plus de vingt ans [Deltombe, Rigouste, in Bancel et al., 2005, p. 191-192]. Ce processus d'essentialisation est particulièrement bien illustré par la construction politico-médiatique de la figure du "garçon arabe " (violent, sexiste, homophobe et antisémite) [Guénif-Souilamas, Macé, 2004]. Cependant, il n'y a pas de reproduction à l'identique : la construction actuelle d'une norme sexuelle nationale illustre un renversement par rapport à la période coloniale, lorsque l'érotisme était du côté de l'Orient. De nos jours, c'est l'inverse : la sexualité opprimée serait du côté de l'Orient et la sexualité libérée du côté de l’Occident [Fassin, 2006].

\section{Dispositifs juridiques et administratifs}

S'inspirant des travaux de Frederick Cooper et Ann L. Stoler [1997] sur les "tensions de l'empire ", plusieurs chercheurs ont critiqué la focalisation sur les représentations pour penser le passé colonial et le présent postcolonial. Ils ont plutôt porté leur attention, d'une part, sur les catégories, les pratiques d'encadrement et les formes d'identification et, d'autre part, sur les dispositions acquises par des agents de l'État dans le contexte colonial. Ils insistent sur le fait que les indépendances produisent des effets au niveau du droit et des statuts : les indigènes sujets de l'empire sont désormais les ressortissants d'un État indépendant, et l'on passe d'une forme de domination bureaucratique à une autre, radicalement différente.

Dans cette perspective, le passé colonial pèse d'abord sur le droit et l'administration des étrangers [Spire, 2005, p. 189-222], en particulier via le statut dérogatoire des Algériens qui, grâce aux accords d'Évian signés en juillet 1962, bénéficient jusqu'en 1967 d'un régime dérogatoire par rapport aux autres étrangers. D'autres ressortissants de pays ex-colonisés (Madagascar, Mali, Sénégal) bénéficient aussi de la liberté de circulation et d'installation, après la signature d'un accord international en juin 1960. Mais, paradoxalement, ce statut privilégié s'articule avec la mise en place de dispositifs spécifiques de contrôle et d'encadrement. C'est ainsi que les services d'assistance technique (SAT), implantés en métropole à partir de 1958 et maintenus quelques années après 1962, s'inspirent de 
l'organisation des sections administratives spécialisées (SAS) chargées du contrôle de la population musulmane en Algérie, et travaillent à la constitution d'un vaste fichier des "Français musulmans ». Suite au nouvel accord franco-algérien de 1967, la liberté de circulation est restreinte par l'obligation de détenir un " certificat de résidence ", qui est l'occasion de constituer le premier fichier national et informatisé d'étrangers. Les pratiques de fichage et de contrôle des Algériens servent ainsi de véritable laboratoire de pratiques bureaucratiques, puisque l'informatisation des titres de séjour est généralisée à tous les étrangers en 1982.

Le droit de la nationalité et les pratiques administratives de naturalisation sont aussi marqués par l'héritage colonial. Ainsi, la procédure d'acquisition de la nationalité par « réintégration » concerne les ex-colonisés, nationaux sans être citoyens [Saada, in Weil, Dufoix, 2005, p. 196-227], qui ont perdu la nationalité française après la décolonisation. L'histoire coloniale ressurgit dès lors que le principe du «double droit du sol » (instauré en 1887, qui déclare français " tout individu né en France d'un étranger qui y est lui-même né ") s'applique aux enfants d'étrangers algériens nés dans un ex-département d'Algérie : ceux-ci deviennent automatiquement français dès leur naissance.

Par ailleurs, les restrictions administratives dans l'application du droit du sol (est déclaré français à sa majorité tout enfant né en France de parents étrangers) à Mayotte révèlent l'instauration d'un véritable « droit postcolonial » [Saada, 2007]. En effet, la grande loi sur la nationalité de 1889, qui instaure le principe du droit du sol dans le droit moderne de la nationalité, était applicable à la métropole, la Guadeloupe, la Martinique, La Réunion et l'Algérie, mais les "Français musulmans d'Algérie » n'étaient pas concernés. C'est une logique discriminatoire analogue qui s'est instaurée à l'encontre des enfants d'origine comorienne reconnus par des citoyens français de Mayotte. Sous prétexte de lutter contre les « reconnaissances fictives », le gouvernement français remet en cause en 2005 l'application du droit du sol dans un territoire d'outre-mer (devenu département d'outre-mer en 2011).

La reproduction d'une logique juridique coloniale après les indépendances s'observe aussi au sujet de la naturalisation des étrangers mariés sous le régime polygamique mais qui vivent en réalité en monogamie. Dans le droit colonial, il fallait renoncer au statut personnel indigène, dont la polygamie, pour prétendre accéder à la citoyenneté française. Dans le contexte postcolonial, jusqu'en 1994, le simple fait que l'acte de mariage des candidats à la naturalisation contienne la mention « mariés sous le régime polygamique " était considéré par l'administration comme un motif de refus de naturalisation (pour « défaut d'assimilation ») : "Une discrimination indirecte et secrète dans les pratiques administratives, fortement contraintes par de nouvelles règles juridiques, se 
substitue à la discrimination directe et légale du droit colonial » [Hajjat, 2012, p. 248].

Une autre manière d'aborder l'héritage colonial consiste à étudier la reconversion des agents de l'État ayant travaillé dans les colonies, en particulier en Algérie, dans l'administration des étrangers en métropole après les indépendances. Les SAT, dirigés par d'anciens militaires de l'armée d'Afrique, sont révélateurs de l'imbrication entre l'action sociale et le contrôle politique [Spire, 2005]. Après 1962, les SAT ne sont pas dissous immédiatement et leurs agents reconvertissent leur savoir-faire dans leur travail d'encadrement social et politique de l'immigration postcoloniale.

D'autres travaux montrent comment des municipalités ont opéré la transition de la catégorie "Français musulman d'Algérie » à celle d' « immigré » et mettent en lumière la construction de compétences en " affaires musulmanes " par des conseilleurs techniques spécialisés en métropole [De Barros, 2006]. Ainsi, d'anciens administrateurs des services civils en Algérie se convertissent après 1962 en conseillers techniques aux affaires musulmanes, rattachés aux préfectures. Dans la même perspective, d'autres travaux montrent comment d'anciens militaires des colonies ont reconverti leur connaissance de la langue arabe et leurs ressources en "mentalité musulmane " pour devenir responsables de camps de harkis [Charbit, 2006] ou directeurs de foyers Sonacotra encadrant les Français musulmans d'Algérie [Hmed, 2006]. Une analyse des trajectoires des hauts fonctionnaires chargés de l'immigration (notamment les énarques) entre 1962 et 1981 permet de conclure à l'absence de « communauté de destin » parmi ceux qui ont connu une expérience algérienne, mais les relations établies pendant la guerre d'Algérie s'imbriquent avec d'autres formes de sociabilité, telles que les affinités politiques, les solidarités de "promo » et les solidarités de corps [Laurens, 2006].

our conclure, on peut définir la notion d'héritage colonial comme un ensemble de ressources symboliques et pratiques construites dans la situation coloniale, notamment des catégories d'entendement, des logiques juridiques et des pratiques administratives, qui sont réactivées dans la situation de l'immigration postcoloniale. Cette réactivation ne correspond pas à une reproduction à l'identique mais dépend d'un nouveau contexte historique, devant se soumettre aux contraintes juridiques du droit postindépendances, et des usages spécifiques qu'en font les acteurs. Cette perspective pourrait nourrir la comparaison entre pays ayant connu une immigration postcoloniale, qui mériterait d'être mieux explorée, dans la mesure où les travaux sur cette question sont rares [Bleich, in Weil, Dufoix, 2005, p. 437-467]. 


\section{Bibliographie}

Bancel N., Bernault F., Blanchard P., Boubeker A., Mbembe A. VERGÈs F. (dir.) (2010), Ruptures postcoloniales, La Découverte, Paris.

Bancel N., Blanchard P., Lemaire S. (dir.) (2005), La Fracture coloniale, La Découverte, Paris.

BAYART I.-F., BERTRAND R. (2006), "De quel "legs colonial" parlet-on? ", Esprit, décembre.

Bertrand R. (2006), Mémoires d'empire, Le Croquant, Bellecombe-en-Bauges.

Blanchard P., Lemaire S. (dir.) (2003), Culture coloniale, Autrement, Paris.

CHARBIT T. (2006), "Un petit monde colonial en métropole ", Politix, $n^{\circ} 76$, p. 31-52.

COOPER F., STOLER A. L. (dir.) (1997), Tensions of Empire, University of California Press, Berkeley.

COQUERY-VIDROVITCH C. (2009), Enjeux politiques de l'histoire coloniale, Agone, Marseille.

DE BARROS F. (2006), "Contours d'un réseau administratif "algérien" et construction d'une compétence en "affaires musulmanes" ", Politix, $n^{\circ} 76$, p. 97-117.

FASSIN É. (2006), « La démocratie sexuelle et le conflit des civilisations ", Multitudes, vol. 3, $n^{\circ} 26$, p. 123-131.

Guénif-SOUILAmas N. (dir.) (2006), La République mise à nu par son immigration, La Fabrique, Paris.

GuÉNif-SOUILAmas N., MacÉ É. (2004), Les Féministes et le Garçon arabe, L'Aube, La Tour-d'Aigues.

Hallat A. (2012), Les Frontières de l'" identité nationale ", La Découverte, Paris.

Hajlat A., Mohammed M. (2013), Islamophobie, La Découverte, Paris.

HMED C. (2006), " "Tenir ses hommes". La gestion des étrangers dans les foyers Sonacotra après la guerre d'Algérie ", Politix, $n^{\circ} 76$, p. $11-30$.

LAURENS S. (2006), « La noblesse d'État à l'épreuve de l'Algérie et de l'après-1962 \#, Politix, n 76, p. 75-96.

LIAUZU C. (2000), « Immigration, colonisation et racisme : pour une histoire liée », Hommes \& Migrations, $n^{\circ} 1228$, p. 5-14.

NOIRIEL G. (2008), Immigration, racisme et antisémitisme en France ( $x \mid x^{e}-x x^{e}$ siècles), Fayard, Paris.

SAADA E. (2007), « Un droit postcolonial », Plein droit, $n^{\circ} 74$.

SAYAD A. (1982), "La naturalisation, ses conditions sociales et sa signification chez les immigrés algériens \# ( $2^{\mathrm{e}}$ partie), GRECO 13 migrations internationales, $n^{\circ} 4-5, p .1-55$. 
SAYAD A. (1994), "Qu'est-ce que l'intégration ? ", Hommes \& Migrations, $n^{\circ} 1182$, p. 8-14.

SMOUTS M.-C. (dir.) (2007), La Situation postcoloniale, Presses de Sciences Po, Paris.

SPIRE A. (2005), Étrangers à la carte, Grasset, Paris.

Weil. P., Dufoix S. (dir.) (2005), L'Esclavage, la colonisation, et après..., PUF, Paris. 